\title{
Mathematical cognition and enculturation: introduction to the Synthese special issue
}

\author{
Markus Pantsar ${ }^{1}$ D
}

Received: 11 November 2019 / Accepted: 13 November 2019 / Published online: 19 November 2019

(c) Springer Nature B.V. 2019

Much theorizing about cognition in philosophy, cognitive science, and biology still proceeds on the assumption that we are born with our primary cognitive faculties pre-determined, and that they simply need to mature, or be fine-tuned by learning mechanisms. Against this view, a growing number of researchers are aligning themselves with the view that processes of enculturation transform our basic biological faculties through the cultural transmission of cognitive practices. In particular, enculturation refers to the transformative process in which interactions with the surrounding culture determine how cognitive practices are acquired and developed (Menary 2015; Fabry 2018a). The enculturation perspective therefore transcends the crude nature versus nurture dichotomy, as it focuses on how the human biological endowment both constrains and allows for specific processes of enculturation to take place. The framework of enculturation has great potential to identify and analyze the integral role of cultural elements in the ontogenetic process of developing cognitive abilities, such as reading, writing, and-of particular interest in this special issue-mathematics.

It is this interplay of biological and cultural elements in the development of human mathematical cognition that provides the background for this Special Issue. During the last decades, a great deal of progress has been made in studying early numerical cognition. Nowadays, it is generally accepted that human infants and many nonhuman animals process observations of their local surroundings in terms of (approximate) quantities. These abilities are thought to be genetically determined adaptations and universal to humans (see, e.g., Dehaene 1997/2011; Butterworth 1999). According to the most commonly accepted theory, this non-symbolic treatment of quantitative information is based on the so-called cognitive core systems (Spelke 2000; Carey

\footnotetext{
With much sadness, we must note that one of the contributors to the Special Issue, Susan Rothstein, died in July 2019, only a few months after her article Quantity evaluations in Yudja: judgements, language and cultural practice (co-authored with Suzi Lima) had appeared online. We are happy and honored to have been able to include Susan's contribution in the Special Issue, which offers a novel and unique perspective on the relationship between mathematics and culture.
}

Markus Pantsar

markus.pantsar@gmail.com

1 University of Helsinki, Helsinki, Finland 
2009). In the literature, two such systems have been identified. First of these is a system for parallel individuation (PIS) (or object tracking, OTS) that allows determining the amount of objects in the field of vision without counting (Starkey and Cooper 1980; Spelke 2000). This ability is called subitizing. The second system allows estimating the numerosity of a group of objects and determining differences in group sizes. It is referred to as the approximate number system (ANS) or the analog magnitude system (Dehaene 1997/2011; Cantlon et al. 2010).

Most researchers agree that the core abilities function as a cognitive basis for the development of arithmetical abilities. However, there is considerable disagreement over the roles that the particular core systems play in the developmental process. Some researchers postulate that the ANS is key to the development of number concepts and arithmetic (e.g. Dehaene 1997/2011; Halberda and Feigenson 2008), while others see parallel individuation as the prevalent core cognitive system in that development (e.g. Carey 2009; Izard et al. 2008; Sarnecka and Carey 2008; Carey et al. 2017; Beck 2017; Cheung and Le Corre 2018). Yet others (Spelke 2011a; Pantsar 2014, 2015; vanMarle et al. 2018) argue that both core cognitive roles play a crucial role in the process.

Amidst this disagreement, however, researchers agree that these core cognitive abilities must not be confused with more advanced (even if still quite elementary) arithmetical abilities. The subitizing ability stops working after three to four objects, and the estimation ability become increasingly inaccurate as the estimated numerosities become larger (see, e.g., Dehaene 1997/2011). To stress this difference, instead of "numerical abilities", which allows for equivocation with more developed abilities, we should call the core cognitive abilities proto-arithmetical (Pantsar 2014, 2018, 2019). ${ }^{1}$ While in the current literature the data on proto-arithmetical cognition is the strongest, similar core cognitive foundations have been proposed also in the case of geometrical cognition (see, e.g., Dehaene et al. 2006; Spelke 2011b).

Putting aside the issue of what exactly constitutes the minimal cognitive starting point [what Heyes (2018) calls the "starter kit"], about which there is still quite some disagreement, the next big question in explaining mathematical cognition then becomes how these proto-mathematical abilities develop into proper mathematical abilities. We believe that the framework of enculturation as formulated by Menary (2015) provides an exceptionally fruitful conceptual framework for studying this question, as it is able to clarify how culturally specific cognitive abilities like mathematics can be acquired and developed taking a particular genetic endowment as a starting point. Through the mechanism that Menary (2014) calls "learning driven plasticity", new cognitive capacities can be acquired due to the neural plasticity of the brain, which allows for both structural and functional variations (Dehaene 2009; Ansari 2008; Anderson 2015). Indeed, the human brain is now thought to be highly plastic, developing in different ways depending on the specific experiences of different individuals (while also being constrained by genetically determined factors). Culturally developed cognitive abilities, such as reading and writing, are thus made possible

\footnotetext{
1 De Cruz et al. (2010) and Pantsar $(2014,2019)$ have argued that it would be clearer if we talked about "numerosities" on the proto-arithmetical level, leaving "numbers" as the domain of more developed arithmetical ability. In the empirical literature, this distinction is rarely made which is the source of some unfortunate formulations, such as Wynn (1992) describing the application of the PIS in infants as "infant arithmetic". Núñez (2017) argues for a similar distinction between "quantical" and "numerical" cognition.
} 
by redeploying older, evolutionarily developed neural circuits for new culturally specific functions (Dehaene 2009; Menary 2014). In the case of arithmetical cognition, a commonly accepted hypothesis is that the evolutionarily determined capacity to code numerosity in the intraparietal sulci is employed in connection with linguistic abilities in grasping number words and symbols, resulting in two different but partially overlapping systems for processing numerosities (Dehaene and Cohen 2007; Nieder and Dehaene 2009; Dehaene 1997/2011; Menary 2015).

The enculturation framework therefore provides a link between the evolutionarily determined, core-cognitive proto-arithmetical abilities and the culturally developed abilities to engage with mathematical concepts and practices. This prompts important questions, since it is well known that there are cultures with little or no basic arithmetic, e.g., the Pirahã and Mundurukú of the Amazon (Gordon 2004; Pica et al. 2004). ${ }^{2}$ Why did these cultures not develop numerical concepts even though they have the same proto-mathematical abilities as we do? To start addressing this question, we must first realize that in cultures where mathematics exists, it is clearly the product of a long line of development. This is consistent with theories of cumulative cultural evolution as the way human cultures develop their knowledge and skill sets (Boyd and Richerson 1985, 2005; Tomasello 1999; Henrich 2015; Heyes 2018). Technologies are improved upon in small generational increments, and in large enough societies - or ones with extensive interactions with other societies - this process can establish a status of knowledge and skills where it is no longer tied to a small group of individuals. Basic arithmetic, for example, developed into such a skill in many cultures. It is typically taught to young children systematically starting from rote learning of the sequence of number words combined with the cognitively more demanding task of counting objects.

Cumulative cultural evolution is a trans-generational process that can help explain how culturally specific cognitive practices develop. The enculturation account can then provide an explanation for how the cultural transmission of these practices happens at the level of individuals, connecting the ontogenetic and phylogenetic study of the development cognitive practices. We can thus construct a model of the development of arithmetical knowledge that is both genetically and culturally determined. Arithmetical ability is partly shaped by the instinctive, core cognitive abilities with quantities that we already possess as newborns, which is then further molded by the training and exposure to practices of counting which in turn are a product of trans-generational cultural accumulation. Due to cultural transmission, in most cultures these abilities are extended to include systems of numerals that refer to small numerosities. At this level, people are able to keep track of small quantities and carry out simple operations (e.g., addition) on them. On some occasions, cultures develop their numeral systems further so that it has a recursive base, which then allows for the unrestricted construction of new numerals, as well as extending the operations for this domain (Pantsar 2018).

An integral part of cumulative cultural evolution in developing arithmetic and other fields of mathematics is the emergence of cognitive tools, such as symbol systems and artifacts like the abacus. Manipulating external symbol systems successfully is

\footnotetext{
2 By "basic arithmetic" we mean the ability to operate (count, add, multiply, etc.) with exact quantities, often in practical settings. This should be distinguished from formal systems of arithmetic, like the Peano axiomatization (see Pantsar 2018, 2019 for more).
} 
integral to learning mathematical practices and thus acquiring mathematical knowledge and skills (Dutilh Novaes 2013). Both domain-specific (abacus, calculator) and multi-domain (pen and paper, computer) cognitive tools shape the way we learn mathematics. This influence also applies to the other direction: mathematical practices are integral to the development of new cognitive tools as can be seen, for example, in the case of calculation practices influencing the development of digital computers (Fabry 2018b). As several researchers have pointed out, many important mathematical problem-solving methods, such as constructing and interpreting diagrams, are made possible by the successful application of tools (see, e.g., De Toffoli and Giardino 2014; Giardino 2017; Fabry and Pantsar 2019). An important part of being enculturated in mathematics is thus being enculturated in the active manipulation of cognitive tools.

The importance of manipulation of cognitive tools prompts the question what kind of cognitive role do we assign to features of our environment, such as artifacts and symbol systems. A natural philosophical setting for studying enculturation is the "4E" landscape of embodied, embedded, enactive, and extended cognition. Menary (2015) places his theory of cognitive integration (CI, Menary 2007) and the enculturation framework in that landscape as a variant of strongly embedded cognition, according to which "cognitive processes and states are integrated with environmental states and processes into a single system" (Menary 2015, p. 2). Menary points out that CI occupies the same "strong embedding region" as extended cognition, according to which some cognitive processes are not entirely located in the brain (Clark and Chalmers 1998; Clark 2008), but distinguishes cognitive integration from the organism-centered approach of Clark, based on the focus on practice and culture that is at the foundation of CI (Menary 2015, pp. 3-4). But what CI and extended cognition have in common is the view that at least some cognitive phenomena are not exclusively constituted by neuronal processes realized in the brain. Based on the significance of number symbols and their manipulation for mathematical cognition, Menary (p. 16) argues:

Symbol manipulation makes a unique difference to our ability to complete mathematical tasks, and we cannot simply ignore their role. If we take the approach of $\mathrm{CI}$, then mathematical cognition is constituted by these bouts of symbol manipulation, and we cannot simply shrink the system back to the brain.

Within this general landscape of proto-arithmetical abilities, enculturation, 4E cognition, and cumulative cultural evolution of mathematical practices and tools, there is a vast range of interesting questions. For this Special Issue, we have gathered contributions that cover a wide range of approaches to enculturation with regard to mathematical cognition. One crucial question concerns how learning driven plasticity is realized on the neuronal level. Menary $(2014,2015)$ follows Dehaene's (2009) theory of neuronal recycling, referring to the way old neural circuits are redeployed to new functions. But Anderson $(2010,2015)$ has proposed a more general principle of neural reuse which, among other differences with respect to the neuronal recycling account, allows for a wider plasticity of regions of the brain for different domains. In this collection, two papers make important contributions to this topic, which we will now see as we introduce the contributions. 
Jones (2018) argues that while neuronal recycling can explain how biological systems can gradually acquire new properties, it is only with the additional mechanisms of neural reuse that we can explain the flexibility of the brain in exploiting these properties to acquire sophisticated culturally shaped abilities like arithmetical problem solving. Fabry (2019) also makes a powerful case for focusing on neural reuse as the mechanism of learning driven plasticity. Furthermore, she argues that the cognitive transformations involved in arithmetical cognition are not limited to the brain. Extending the cognitive transformations to the extra-cerebral bodily counterpart of learning driven plasticity, which she calls learning driven bodily adaptability (Fabry 2018a), Fabry uses examples like finger counting and embodied symbol manipulation to show that the adaptability of our motor patterns play an integral role in acquiring arithmetical knowledge and skills in socio-cultural settings. Finally, in her paper the enculturation account is strengthened by an analysis of disorders in arithmetical cognition, namely developmental dyscalculia and acquired acalculia.

In their article, Johansen and Misfeldt (2018) analyze the embodied physical experiences in practices of mathematics based on an interview study of active research mathematicians. Their analysis lends support to the position that it is crucial to include social and cultural contexts when analyzing the cognitive use of material representations such as diagrams by mathematicians. In particular, they argue that material representations and the ways mathematicians engage with them are socially sanctioned and enabled in a process of enculturation. Larvor (2018) also tackles the issue of material representations in his paper. He uses examples from algebra to argue that the application of notations and representations is a dynamic process which is integral to mathematical cognition, shaping the way mathematics itself develops. Larvor argues that the common parlance of material tools as "scaffolding" is in fact misleading in that it suggests that notations and representations are static aids that can be discarded later. Indeed, he argues that much of mathematics, and consequently the cognitive tasks involved in it, would not even exist without those notations and representations, which continue to play a crucial role in these cognitive processes and thus cannot be discarded at a later stage.

This is in line with what Vold and Schlimm (2019) argue in their contribution to this volume. They use examples from the history of mathematics in which introducing new symbols has enabled representing mathematical possibilities that were not yet understood or conceived (e.g. negative numbers), but also entirely new domains (like non-Euclidean geometries). They thus argue that symbols can drive mathematical understanding in crucial ways, and so extra-neural representations like symbols can have non-derived, "original" content. This issue is crucial when we consider just what "cognition" consists of in the enculturation framework. In his paper, Buijsman (2018) approaches this question with focus on 4E cognition and where Menary's enculturation account falls on that landscape. He analyses the role of numerals in developing arithmetical cognition and asks whether the role they play indeed implies that the cognitive process cannot be "shrunk" back to the brain. While he ends up accepting that the cognitive role of symbol manipulation requires the endorsement of some form of externalism, he argues that the empirical data on arithmetical cognition do not give us sufficient reasons to prefer a theory of extended (or strongly embedded) cognition over a weaker of theory of embedded cognition. According to the latter, 
interactions with the environment play an important role in cognition but cognitive processes do not extend beyond our heads to objects in the environment (Sprevak 2010).

While Buijsman's analysis of Menary's theory focuses on the suitable theory on the 4E cognition spectrum for understanding enculturation, Pelland (2019) argues that the enculturation account may not have the generality that Menary aimed at. In particular, Pelland claims that, while enculturation may help explain differences between cultures that have developed numerical abilities and cultures that do not, it does not provide an explanation of the difference between individuals with and without developed numerical abilities. In this way, he argues that the enculturation account is more suitable as an account of transmission of mathematical practices rather than as an account of mathematical innovation.

This general question of how enculturation can account for innovation is most certainly crucial. But when applying the enculturation account to analyze mathematical innovation and practices, the devil is very much in the details. In this Special Issue, two papers are included that study particular innovations and practices. Barton (2018) proposes an explanation of how the metaphysics and epistemology of zero can be analyzed satisfactorily within the enculturation framework by combining mathematical (numbers as properties of collections), philosophical (the philosophy of absence), and empirical (numerical cognition) studies. Rothstein and Lima (2018) present a study of the interpretation of quantity expressions in the Amazonian language of Yudja. The Yudja language allows for reference to exact cardinalities of collections, but Rothstein and Lima show that the expressions of exact cardinalities are not automatically used for exact measurements. They thus argue that in order to account for knowledge and skills in the domain of measurements, the process requires more than the enculturated ability to perform abstract calculations.

Finally, it is important to note that while successful processes of enculturation are integral the acquisition of new cognitive practices, enculturation is always tied to beliefs shared in a particular cultural context. Differently enculturated individuals and groups may struggle when assimilating to new cultures, and mathematics is no exception. In the final paper of the Special Issue, Rittberg et al. (2018) apply the recent philosophical work on epistemic injustice (Fricker 2007; Kidd and Pohlhaus 2017) to mathematics. They present cases from the history of mathematics (most importantly Thomas Royen and Srinivasa Ramanujan) and analyze how processes of enculturation can lead to epistemic injustice by hindering the participation of certain individuals in mathematical cultures. Their contribution highlights that cultural factors can also represent barriers for the development of mathematical knowledge, that is, when certain individuals are not attributed the epistemic stature they deserve in virtue of non-epistemic factors such as gender, race, nationality etc.

This Special Issue was inspired by the Mathematical Cognition and Enculturation Symposium at the meeting of the European Society of Philosophy and Psychology in St. Andrews in 2016, organized by Catarina Dutilh Novaes. Two of the papers in this volume (by Max Jones and Jean-Charles Pelland) are based on talks given in that symposium. I would like to express my greatest gratitude to Professor Dutilh Novaes for her work as the co-editor of this volume, as well as for her help in writing this introduction. Both the symposium and this Special Issue were strongly influenced by 
the work of Richard Menary on enculturation and mathematical cognition. We would like to express our greatest gratitude for his central role in making this Special Issue possible. $^{3}$

\section{References}

Anderson, M. L. (2010). Neural reuse: A fundamental organizational principle of the brain. Behavioral and Brain Sciences, 33(4), 245-266.

Anderson, M. (2015). After phrenology: Neural reuse and the interactive brain. Cambridge, MA: MIT Press.

Ansari, D. (2008). Effects of development and enculturation on number representation in the brain. Nature Reviews Neuroscience, 9(4), 278-291.

Barton, N. (2018). Absence perception and the philosophy of zero. Synthese. https://doi.org/10.1007/s112 29-019-02220-x.

Beck, J. (2017). Can bootstrapping explain concept learning? Cognition, 158, 110-121.

Boyd, R., \& Richerson, P. J. (1985). Culture and the evolutionary process. Chicago: University of Chicago Press.

Boyd, R., \& Richerson, P. J. (2005). Not by genes alone. Chicago: University of Chicago Press.

Buijsman, S. (2018). How numerals support new cognitive capacities. Synthese. https://doi.org/10.1007/s1 1229-018-01989-7.

Butterworth, B. (1999). What counts: How every brain is hardwired for math. New York: The Free Press.

Cantlon, J. F., Safford, K. E., \& Brannon, E. M. (2010). Spontaneous analog number representations in 3-year-old children. Developmental Science, 13(2), 289-297.

Carey, S. (2009). The origin of concepts. New York: Oxford University Press.

Carey, S., Shusterman, A., Haward, P., \& Distefano, R. (2017). Do analog number representations underlie the meanings of young children's verbal numerals? Cognition, 168, 243-255.

Cheung, P., \& Le Corre, M. (2018). Parallel individuation supports numerical comparisons in preschoolers. Journal of Numerical Cognition, 4(2), 380-409.

Clark, A. (2008). Supersizing the mind: Embodiment, action, and cognitive extension. Oxford: Oxford University Press.

Clark, A., \& Chalmers, D. (1998). The extended mind. Analysis, 58(1), 7-19.

De Cruz, H., Neth, H., \& Schlimm, D. (2010). The cognitive basis of arithmetic (pp. 59-106). Konstanz: Bibliothek der Universität Konstanz.

De Toffoli, S., \& Giardino, V. (2014). Forms and roles of diagrams in knot theory. Erkenntnis, 79(4), $829-842$.

Dehaene, S. (1997/2011). The number sense: How the mind creates mathematics, 2nd edition. New York: Oxford University Press.

Dehaene, S. (2009). Reading in the brain: The new science of how we read. London: Penguin.

Dehaene, S., \& Cohen, L. (2007). Cultural recycling of cortical maps. Neuron, 56(2), 384-398.

Dehaene, S., Izard, V., Pica, P., \& Spelke, E. (2006). Core knowledge of geometry in an Amazonian indigene group. Science, 311(5759), 381-384.

Dutilh Novaes, C. (2013). Mathematical reasoning and external symbolic systems. Logique et Analyse, 56(221), 45-65.

Fabry, R. E. (2018a). Betwixt and between: The enculturated predictive processing approach to cognition. Synthese, 195(6), 2483-2518.

Fabry, R. E. (2018b). Turing redux: Enculturation and computation. Cognitive Systems Research, 52, $793-808$.

Fabry, R. E. (2019). The cerebral, extra-cerebral bodily, and socio-cultural dimensions of enculturated arithmetical cognition. Synthese. https://doi.org/10.1007/s11229-019-02238-1.

Fabry, R. E., \& Pantsar, M. (2019). A fresh look at research strategies in computational cognitive science: The case of enculturated mathematical problem solving. Synthese. https://doi.org/10.1007/s11229-01 9-02276-9.

\footnotetext{
3 Parts of this introduction are adapted from Pantsar (2019), which is acknowledged with gratitude to Frontiers in Psychology.
} 
Fricker, M. (2007). Epistemic injustice: Power and the ethics of knowing. Oxford: Oxford University Press.

Giardino, V. (2017). Diagrammatic reasoning in mathematics. In L. Magnani \& T. Bertolotti (Eds.), Springer handbook of model-based science (pp. 499-522). Heidelberg: Springer.

Gordon, P. (2004). Numerical cognition without words: Evidence from Amazonia. Science, 306, 496-499.

Halberda, J., \& Feigenson, L. (2008). Set representations required for acquisition of the natural number concept. Behavioral and Brain Sciences, 31(6), 655-656.

Henrich, J. (2015). The secret of our success: How culture is driving human evolution, domesticating our species, and making us smarter. Princeton University Press.

Heyes, C. (2018). Cognitive gadgets: The cultural evolution of thinking. Cambridge: Harvard University Press.

Izard, V., Pica, P., Spelke, E. S., \& Dehaene, S. (2008). Exact equality and successor function: Two key concepts on the path towards understanding exact numbers. Philosophical Psychology, 21(4), 491-505.

Johansen, M. W., \& Misfeldt, M. (2018). Material representations in mathematical research practice. Synthese. https://doi.org/10.1007/s11229-018-02033-4.

Jones, M. (2018). Numerals and neural reuse. Synthese. https://doi.org/10.1007/s11229-018-01922-y.

Kidd, I. J., \& Pohlhaus, J. G. (Eds.). (2017). The Routledge handbook of epistemic injustice. London: Taylor \& Francis.

Larvor, B. (2018). Why 'scaffolding' is the wrong metaphor: The cognitive usefulness of mathematical representations. Synthese, 1-14.

Menary, R. (2007). Cognitive integration: Mind and cognition unbounded. London: Palgrave Macmillan.

Menary, R. (2014). Neuronal recycling, neural plasticity and niche construction. Mind and Language, 29(3), $286-303$.

Menary, R. (2015). Mathematical cognition: A case of enculturation. Frankfurt am Main: Open MIND, MIND Group.

Nieder, A., \& Dehaene, S. (2009). Representation of number in the brain. Annual Review of Neuroscience, 32, 185-208.

Núñez, R. E. (2017). Is there really an evolved capacity for number? Trends in Cognitive Sciences, 21(6), $409-424$.

Pantsar, M. (2014). An empirically feasible approach to the epistemology of arithmetic. Synthese, 191(17), 4201-4229.

Pantsar, M. (2015). In search of aleph-null: How infinity can be created. Synthese, 192(8), 2489-2511.

Pantsar, M. (2018). Early numerical cognition and mathematical processes. Theoria, 33(2), 285-304.

Pantsar, M. (2019). The enculturated move from proto-arithmetic to arithmetic. Frontiers in Psychology, $10,1454$.

Pelland, J. C. (2019). What's new: Innovation and enculturation of arithmetical practices. Synthese, 1-26.

Pica, P., Lemer, C., Izard, V., \& Dehaene, S. (2004). Exact and approximate arithmetic in an Amazonian indigene group. Science, 306(5695), 499-503.

Rittberg, C. J., Tanswell, F. S., \& Van Bendegem, J. P. (2018). Epistemic injustice in mathematics. Synthese. https://doi.org/10.1007/s11229-018-01981-1.

Rothstein, S., \& Lima, S. (2018). Quantity evaluations in Yudja: Judgements, language and cultural practice. Synthese. https://doi.org/10.1007/s11229-018-02016-5.

Sarnecka, B. W., \& Carey, S. (2008). How counting represents number: What children must learn and when they learn it. Cognition, 108(3), 662-674.

Spelke, E. S. (2000). Core knowledge. American psychologist, 55(11), 1233.

Spelke, E. S. (2011a). Quinean bootstrapping or Fodorian combination? Core and constructed knowledge of number. Behavioral and Brain Sciences, 34, 149-150.

Spelke, E. (2011b). Natural number and natural geometry. In Dehaene \& Brannon (Eds.), Space, time and number in the brain (pp. 287-318). London: Academic Press.

Sprevak, M. (2010). Inference to the hypothesis of extended cognition. Studies in History and Philosophy of Science Part A, 41(4), 353-362.

Starkey, P., \& Cooper, R. G. (1980). Perception of numbers by human infants. Science, 210(4473), 1033-1035.

Tomasello, M. (1999). The cultural origins of human cognition. Cambridge, MA: Harvard University Press. vanMarle, K., Chu, F. W., Mou, Y., Seok, J. H., Rouder, J., \& Geary, D. C. (2018). Attaching meaning to the number words: Contributions of the object tracking and approximate number systems. Developmental Science, 21(1), e12495. 
Vold, K., \& Schlimm, D. (2019). Extended mathematical cognition: External representations with nonderived content. Synthese. https://doi.org/10.1007/s11229-019-02097-w.

Publisher's Note Springer Nature remains neutral with regard to jurisdictional claims in published maps and institutional affiliations. 